\title{
Accuracy of parameter estimation of gravitational waves with LISA
}

\author{
Michael Peterseim, Oliver Jennrich and Karsten Danzmann \\ Universität Hannover, Appelstrasse 2, D-30167 Hannover, Germany
}

\begin{abstract}
LISA is a space-borne, laser-interferometric gravitational-wave detector currently under study by the European Space Agency. We give a brief introduction about the main features of the detector, concentrating on its one-year orbital motion around the Sun. We compute how the amplitude as well as the phase of a gravitational wave are modulated due to this motion by transforming an arbitrary gravitational-wave signal in a reference frame that is rigidly fixed to the arms of the detector. To see how LISA works the detector response to a gravitational wave which is purely monochromatic in the barycentric frame will be discussed.

A brief review of the theory of parameter estimation, based on the work of Finn and Cutler, will be given. Following this theory the detection of a gravitational-wave signal buried in detector noise was simulated numerically. We interpret the results of this simulation to determine the angular resolution of LISA.
\end{abstract}

PACS number: $0480 \mathrm{~N}$

\section{Introduction}

The LISA mission consists of six spacecraft forming a laser interferometer in a plane inclined $60^{\circ}$ with respect to the ecliptic and along a circle with a radius of $3 \times 10^{9} \mathrm{~m}$ [1]. Two spacecraft will be placed at each of three points on this circle forming an equilateral triangle with baselines of $5 \times 10^{9} \mathrm{~m}$ which rotates clockwise around its centre, as viewed from the Sun, with a period of one year. The complete constellation describes an approximately circular orbit at a distance of $R=1 \mathrm{AU}$ from the Sun and trailing the Earth in its orbit by $20^{\circ}$. As a single, nonmoving detector reveals no information about the directional parameters of the source of the gravitational wave, all the information about the source parameters is contained in the variation of the detector response that results from the described motion [5].

Firstly, the detected amplitude of the gravitational wave is modulated, as the detector's sensitivity pattern is not isotropic, so the detected amplitude depends on the location of the source relative to the detector and this location changes due to the rotation of the detector formation around its centre.

Secondly, the detector is moving relative to the source due to the periodic motion of its centre along its orbit around the Sun, Doppler-shifting the frequency of the gravitational wave. This results in a phase modulation of the detector output.

The amplitude modulation as well as the phase modulation will spread a sharply peaked monochromatic signal into a set of sidebands separated from the carrier by integer multiples of the fundamental frequency, $(1 \text { year })^{-1}$. In the following sections both modulations will be calculated and a review of some standard techniques of parameter estimation will be given. Finally, these techniques are applied to the simulated data for a linearly polarized, monochromatic wave at a frequency of $3 \mathrm{mHz}$ and interpreted to give information about the potential accuracy of the estimation of the angular parameters of the source. 


\section{The amplitude modulation}

The amplitude modulation can be calculated by transforming the metric tensors

$$
\boldsymbol{h}_{\times}:=h_{\times}\left(\begin{array}{lll}
0 & 1 & 0 \\
1 & 0 & 0 \\
0 & 0 & 0
\end{array}\right) \quad \text { and } \quad \boldsymbol{h}_{+}:=h_{+}\left(\begin{array}{rrr}
1 & 0 & 0 \\
0 & -1 & 0 \\
0 & 0 & 0
\end{array}\right)
$$

which are defined in the source frame, i.e. a system with its $x$-axis in the $x-y$-plane of the barycentric frame, its $z$-axis pointing towards the Sun and the source at its origin. The transformation is split into one transforming the source system into the barycentric system and another one from the barycentric frame into the detector frame, which is rigidly fixed to the interferometer arms.

Let $\theta$ and $\phi$ be the Euler angles that define the source position in the barycentric frame, with its $x-y$-plane in the ecliptic (cf figure 1). The transformation into the source system is composed of two rotations. The first, realized by the rotation matrix $\boldsymbol{a}_{1}$, turns the $y$-axis of the barycentric frame on the projection of the line connecting the Sun and the source on the ecliptic, that is counterclockwise through an angle $\phi-90^{\circ}$ around the $z$-axis,

$$
a_{1}:=\left(\begin{array}{ccc}
\sin \phi & -\cos \phi & 0 \\
\cos \phi & \sin \phi & 0 \\
0 & 0 & 1
\end{array}\right)
$$

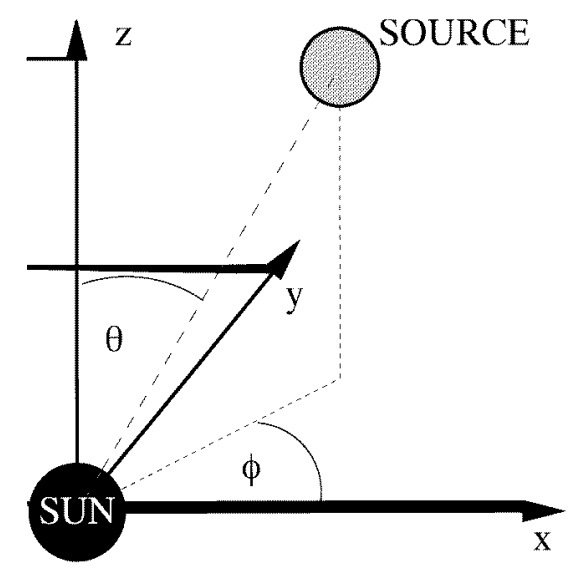

Figure 1. Orientation of the source in the barycentric frame.

A second rotation $b_{1}$ turns the system counterclockwise around the new $x$-axis by $180^{\circ}-\theta$. With $\boldsymbol{T}_{1}:=\boldsymbol{b}_{1} \boldsymbol{a}_{1}$ the matrix $\boldsymbol{h}_{+}$of (1) is transformed from the source system into the barycentric frame by

$$
h_{+} \rightarrow \boldsymbol{T}_{1}^{\mathrm{t}} \boldsymbol{h}_{+} \boldsymbol{T}_{1} .
$$

The following angles are used to calculate the transformation into the detector system:

$$
\psi_{\mathrm{a}}:=2 \frac{\pi t}{T} \quad \psi_{\mathrm{b}}:=\frac{1}{3} \pi \quad \psi_{\mathrm{c}}:=-2 \frac{\pi t}{T}+\alpha
$$

where $\frac{1}{3} \pi$ is the angle of LISA with respect to the ecliptic [1] and $\alpha$ is the phase between LISA's motion around the Sun and the motion around its centre of mass.

Now a rotation matrix $\boldsymbol{a}_{2}$ turns the frame of reference in the barycentric system counterclockwise around the $z$-axis by $\psi_{\mathrm{a}}$, so that the new $y$-axis points towards LISA. 
Then $\boldsymbol{b}_{2}$ turns it by $\psi_{\mathrm{b}}$ out of the ecliptic. Finally, $\boldsymbol{c}_{2}$ turns it clockwise around the new $z$-axis by $\psi_{\mathrm{c}}$. A vector is transformed from the barycentric into the detector system by $\boldsymbol{T}_{2}=\boldsymbol{c}_{2} \boldsymbol{b}_{2} \boldsymbol{a}_{2}$. Therefore the matrix $\boldsymbol{h}_{+}$is transformed as

$$
h_{+} \rightarrow \underbrace{T_{2} T_{1}^{\mathrm{t}}}_{=: T} h_{+} T_{1} T_{2}^{\mathrm{t}}
$$

\section{The phase modulation}

The translatory motion of the detector relative to the source leads to a phase modulation of the gravitational-wave signal. This modulation can be easily calculated with the so-called barycentric transform between time of arrival at the Solar System and time at the detector [2]. In the former system, which can be considered to be a convenient inertial frame, the signal is not modulated at timescales short or comparable to an Earth orbit and therefore is of fixed frequency. Let $s_{\mathrm{d}}$ and $s_{\mathrm{b}}$ be the signals at the detector and at the barycentre, respectively, then by definition

$$
s_{\mathrm{d}}\left(t_{\mathrm{d}}\right)=s_{\mathrm{b}}\left(t_{\mathrm{b}}\left[t_{\mathrm{d}}, \theta, \phi\right]\right),
$$

where $(\theta, \phi)$ is the angular position of the source (cf figure 1). The relation between the two time variables $t_{\mathrm{b}}, t_{\mathrm{d}}$ is given by

$$
t_{\mathrm{b}}\left[t_{\mathrm{d}}, \theta, \phi\right]=t_{\mathrm{d}}+\frac{\vec{n}(\theta, \phi) \vec{d}\left(t_{\mathrm{d}}\right)}{c}
$$

with $\vec{n}$ being a unit vector pointing towards the source and $\vec{d}$ a vector connecting LISA and the solar system, so it has a length of $R=1 \mathrm{AU}$ :

$$
\vec{n}=\left(\begin{array}{c}
\cos \phi \sin \theta \\
\sin \phi \sin \theta \\
\cos \theta
\end{array}\right) \quad \vec{d}=R\left(\begin{array}{c}
\sin (2 \pi t / T) \\
\cos (2 \pi t / T) \\
0
\end{array}\right) .
$$

Therefore the relation between the two signals $s_{\mathrm{d}}$ and $s_{\mathrm{b}}$ as functions of time is

$$
s_{\mathrm{d}}\left(t_{\mathrm{d}}\right)=s_{\mathrm{b}}\left(t_{\mathrm{d}}+\frac{R \sin \theta}{c} \cos \left(\frac{2 \pi t}{T}-\phi\right)\right) .
$$

So if the signal in the inertial frame is purely sinusoidal of frequency $f_{\mathrm{GW}}$, in the detector response it appears as

$$
\begin{aligned}
s_{\mathrm{d}}\left(t_{\mathrm{d}}\right) & =\sin \left(2 \pi f_{\mathrm{GW}} t_{\mathrm{b}}\right) \\
& =\sin (2 \pi f_{\mathrm{GW}} t_{\mathrm{d}}+\underbrace{\frac{2 \pi f_{\mathrm{GW}} R \sin \theta}{c} \cos \left(\frac{2 \pi t}{T}-\phi\right)}_{\Phi(t)}),
\end{aligned}
$$

including a phase modulation $\Phi(t)$ with a modulation index $m$ of

$$
\begin{aligned}
m & =\frac{2 \pi f_{\mathrm{GW}} R \sin \theta}{c} \\
& \approx \pi \sin \theta\left(\frac{f_{\mathrm{GW}}}{1 \mathrm{mHz}}\right) .
\end{aligned}
$$




\section{The LISA response to a gravitational wave}

A gravitational wave which is purely sinusoidal in the barycentric frame causes a strain perceived by the detector given by

$\boldsymbol{H}=\boldsymbol{T}\left(\begin{array}{cccc}0 & 0 & 0 & 0 \\ 0 & h_{p l} & h_{k r} & 0 \\ 0 & -h_{k r} & h_{p l} & 0 \\ 0 & 0 & 0 & 0\end{array}\right) \boldsymbol{T}^{\mathrm{t}} \exp \left\{\mathrm{i}\left[2 \pi f_{\mathrm{GW}} t-\vec{k}_{\mathrm{GW}} \vec{x}+\Phi(t)\right]\right\}$

with the phase modulation $\Phi(t)$ of equation (10) and the modulated amplitude $\boldsymbol{T}\left(\boldsymbol{h}_{+}+\boldsymbol{h}_{\times}\right) \boldsymbol{T}^{\mathrm{t}}$ (cf equation (5)). To see how the gravitational-wave detector works, recall that general relativity predicts that a ray of light connects a set of points by an interval of zero or

$$
\mathrm{d} s^{2}=0 .
$$

For simplicity, one of the detector arms is chosen to give the $x$-axis of the detector frame, while the other arm points to the 'first quadrant' at an angle of $\alpha=60^{\circ}$ to the $x$-axis. The above equation then becomes

$$
\begin{aligned}
0= & \mathrm{d} s^{2} \\
= & g_{\mu \nu} \mathrm{d} x^{\mu} \mathrm{d} x^{\nu} \\
= & -c^{2} \mathrm{~d} t^{2}+\left(1+H_{11}(t, \vec{x})\right) \mathrm{d} x^{2}+\left(1+H_{22}(t, \vec{x})\right) \mathrm{d} y^{2}+H_{12}(t, \vec{x}) \mathrm{d} x \mathrm{~d} y \\
& +H_{21}(t, \vec{x}) \mathrm{d} y \mathrm{~d} x \\
= & -c^{2} \mathrm{~d} t^{2}+\left[\left(1+H_{11}(s)\right) \cos ^{2} \alpha+\left(1+H_{22}(s)\right) \sin ^{2} \alpha\right. \\
& \left.+\left(H_{12}(s)+H_{21}(s)\right) \sin \alpha \cos \alpha\right] \mathrm{d} s^{2}
\end{aligned}
$$

where the parameter $s$ is the parametrization of the trajectory and $\alpha$ equals $60^{\circ}$ for one arm of the interferometer and $0^{\circ}$ for the other. This means that the gravitational wave modulates the light travel time $\tau_{t t}$ between two neighbouring points of fixed coordinate separation which can be calculated by integrating the square root of the above equation

$$
\begin{aligned}
c \int_{0}^{\tau_{t t}} \mathrm{~d} t=\int_{0}^{L} \sqrt{1+H_{11}(s) \cos ^{2} \alpha+H_{22}(s) \sin ^{2} \alpha+\frac{1}{2}\left(H_{12}(s)+H_{21}(s)\right) \sin 2 \alpha} \mathrm{d} s \\
\quad-\int_{L}^{0} \sqrt{1+\cdots} \mathrm{d} s,
\end{aligned}
$$

where $L$ is the interferometer arm length. Assuming that the metric perturbation is approximately constant during the time any given wavefront is present in the apparatus (i.e. $f_{\mathrm{GW}} \tau_{t t} \ll 1$ ), and expanding the root, leads to a difference in light travel time for the interferometer arms of

$$
\Delta \tau_{t t}=\frac{2 L}{8 c} \sqrt{3}\left[\left(H_{22}-H_{11}\right) \sqrt{3}+H_{12}+H_{21}\right]
$$

and, taking $\lambda$ to be the laser wavelength, a corresponding measurable phase difference of

$$
\Delta \Phi=\frac{2 L \pi}{4 \lambda} \sqrt{3}\left[\left(H_{22}-H_{11}\right) \sqrt{3}+H_{12}+H_{21}\right]
$$

where $\boldsymbol{H}$ still has the same time dependence as in (12). Due to the motion of the detector a signal that is monochromatic in the source system will now be spread into a set of 
sidebands in the detector system that contains all the information about the parameters of the gravitational wave. The resulting line spectra for two source locations are presented in figures 2 and 3. The frequency of the gravitational wave in the example given in the figures is $3 \mathrm{mHz}$, and the source locations are $(\theta, \phi)=(\pi / 2,0)$ or $(\theta, \phi)=(\pi / 4,0)$ respectively.
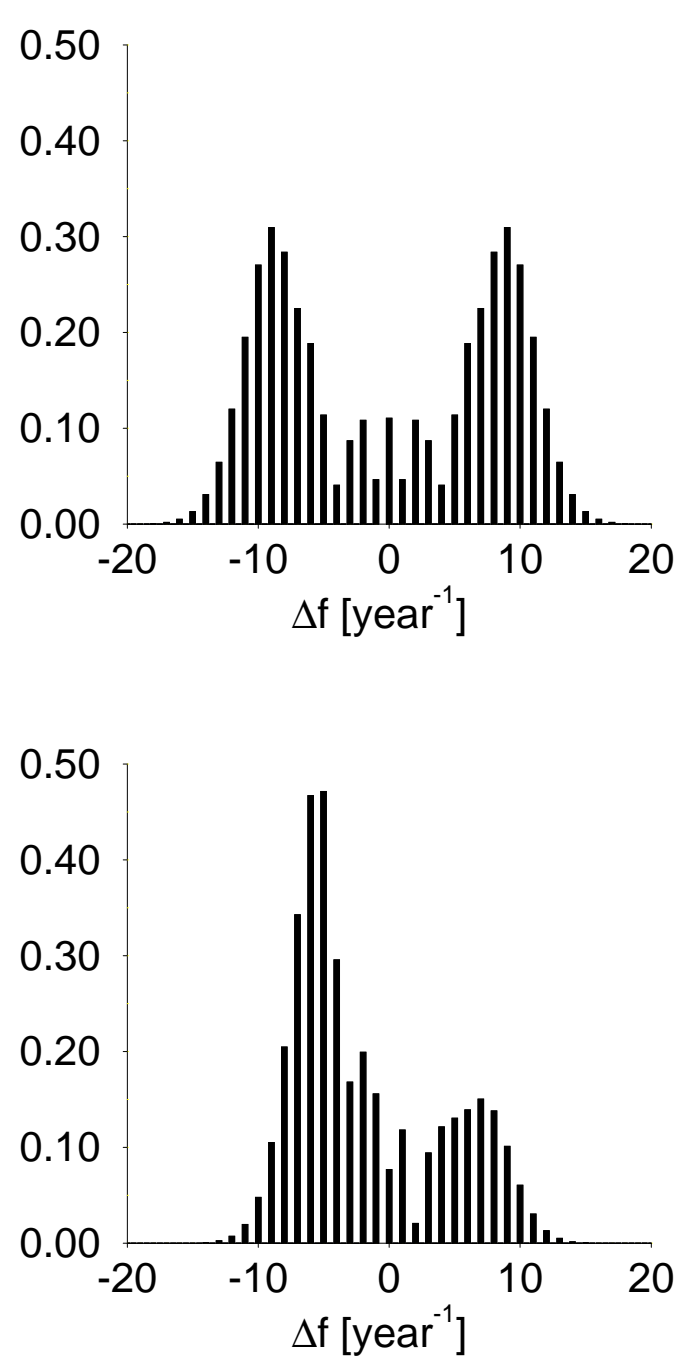

Figure 2. Sideband structure of the detector response to a monochromatic, pluspolarized gravitational wave at $3 \mathrm{mHz}$, corresponding to a modulation index of $m \approx 10$ (cf equation (11)). The source is located at $(\theta, \phi)=(\pi / 2,0)$.

Figure 3. Sideband structure of the detector response to a monochromatic, pluspolarized gravitational wave at $3 \mathrm{mHz}$, corresponding to a modulation index of $m \approx 10$ (cf equation (11)). The source is located at $(\theta, \phi)=(\pi / 4,0)$.

\section{Review of parameter estimation}

The problem of measurement is to determine the values of some or all parameters of the signal [3]. It will be shown how accurately that can be done. Consider a stream $s(t)$ that represents the pure detector output $h(\vec{\mu})$, parametrized by several unknown parameters $\mu_{i}$ collectively denoted as $\vec{\mu}=\left(\mu_{1}=\theta, \mu_{2}=\phi, \ldots\right)$ plus additional noise $n(t)$. Now one has to find a probability density function $P(\vec{\mu}, s)$ for the parametrization $\vec{\mu}$ that characterizes the detector output $h(\vec{\mu})$. Assuming that $n(t)$ is a Gaussian process with zero mean, 
characterized by the one-sided power spectral density $S_{n}(f)$, it can be shown [4] that

$$
P(\vec{\mu}, s) \sim \exp \langle s, h(\vec{\mu})\rangle
$$

where the symmetric inner product is defined as

$$
\langle s, h\rangle=2 \int_{0}^{\infty} \frac{s(f) h^{*}(f)+h(f) s^{*}(f)}{S_{n}(f)} \mathrm{d} f .
$$

From this definition it follows that, for a waveform $h(\vec{\mu})$, the signal-to-noise ratio is given approximately by

$$
\frac{S}{N}[h(\vec{\mu})]=\frac{\langle h(\vec{\mu}), h(\vec{\mu})\rangle}{\operatorname{RMS}(\langle\mathrm{h}(\vec{\mu}), \mathrm{n}\rangle)}=\sqrt{\langle h(\vec{\mu}), h(\vec{\mu})\rangle} .
$$

The error in measurement is taken to be the width of the probability density function $P(\vec{\mu}, s)$ for the measured value $\hat{\vec{\mu}}$, i.e. the variance-covariance matrix

$$
\Sigma_{i j}=\int\left(\mu_{i}-\hat{\mu}_{i}\right)\left(\mu_{j}-\hat{\mu}_{j}\right) P(\vec{\mu}, s) \mathrm{d}^{n} \mu .
$$

There are several choices of data-processing algorithms to find $\hat{\vec{\mu}}$; one is the so-called Bayes estimator $\hat{\vec{\mu}}_{\text {Bayes }}=\int \vec{\mu}_{i} P(\vec{\mu}, s) \mathrm{d}^{n} \mu$.

\section{Results}

In table 1 RMS errors are presented as square roots of $\Sigma_{\theta \theta}$ and $\Sigma_{\phi \phi}$ that have been calculated for a monochromatic, linearly plus-polarized wave at $3 \mathrm{mHz}$ with constant amplitude over one year of observation. Only values of $\theta<\pi / 2$ and $\phi=0$ appear in that table, because the errors are symmetrical in $\pi / 2 \pm \theta$ and in $\phi$. For a signal-to-noise ratio of 100 the position accuracy comes out as $1-10 \mathrm{mrad}$, depending on the value of $\theta$.

Table 1. The RMS errors for the angular parameters $\theta$ and $\phi$ (cf figure 1 for $\phi=0$ and a signal-to-noise ratio of 115 , that put a constraint on the angular position accuracy of $10^{-6} \mathrm{sr}$ ).

\begin{tabular}{llll}
\hline Location $(\mathrm{rad})$ & $\delta \theta(\mathrm{mrad})$ & $\delta \phi(\mathrm{mrad})$ & $\mathrm{d} \Omega=\sin \theta \delta \theta \delta \phi\left(\mathrm{mrad}^{2}\right)$ \\
\hline$\theta=0.10 \pi$ & 1.99 & 5.58 & 3.10 \\
$\theta=0.15 \pi$ & 2.33 & 3.65 & 3.87 \\
$\theta=0.20 \pi$ & 2.79 & 2.84 & 4.64 \\
$\theta=0.25 \pi$ & 3.27 & 2.43 & 5.45 \\
$\theta=0.30 \pi$ & 3.92 & 2.08 & 6.47 \\
$\theta=0.35 \pi$ & 5.52 & 1.88 & 9.06 \\
$\theta=0.40 \pi$ & 6.81 & 1.69 & 10.82 \\
$\theta=0.45 \pi$ & 9.27 & 1.59 & 14.38 \\
$\theta=0.50 \pi$ & 10.94 & 1.57 & 16.55 \\
\hline
\end{tabular}

\section{References}

[1] Danzmann K et al 1993 Proposal for a laser-interferometric gravitational wave detector in space Report $M P Q$ 177 Max-Planck-Institut für Quantenoptik

[2] Schutz B F 1991 The Detection of Gravitational Waves ed D Blair pp 406-52

[3] Cutler C and Flanagan E 1994 Phys. Rev. D 492658

[4] Finn L S 1992 Phys. Rev. D 465236

[5] Saulson P R 1994 Fundamentals of Interferometric Wave Detectors (Singapore: World Scientific) 